\title{
Measuring Non-motorized Accessibility: Issues, Alternatives, and Execution
}

\author{
Michael Iacono* ${ }^{1}$ \\ Research Fellow, Department of Civil Engineering, University of Minnesota \\ 500 Pillsbury Drive SE \\ Minneapolis, MN, USA 55455 \\ Phone: 612-626-0024, Fax: 612-626-7750 \\ iaco0009@umn.edu \\ Kevin J. Krizek ${ }^{2}$ \\ Associate Professor of Planning, Design, and Civil Engineering, University of Colorado \\ P.O. Box 173364, Denver, Colorado 80217 \\ Phone: 303-556-3282, Fax: 303-492-6163 \\ krizek@colorado.edu \\ Ahmed El-Geneidy \\ Assistant Professor, School of Urban Planning, McGill University \\ Suite 401, McDonald-Harrington Building \\ 815 Sherbrooke Street West \\ Montreal, Quebec, Canada H3A 2K6 \\ Phone: 514-398-8741, Fax: 514-398-8376 \\ ahmed.elgeneidy@mcgill.ca \\ *Corresponding author
}

Please cite as Iacono, M., Krizek, K., \& El-Geneidy, A. (2010). Measuring non-motorized accessibility: Issues, alternatives, and execution. Journal of Transport Geography, 18, 133-140.

\footnotetext{
${ }^{1}$ Nexus Research Group, University of Minnesota

${ }^{2}$ Director, Active Communities / Transportation (ACT) Research Group
} 


\begin{abstract}
While the transportation planning literature contains many examples of the calculation of measures of accessibility for urban areas, these measures are largely restricted to motorized modes and to a handful of destination activities. This paper explores the issues related to the development of accessibility measures for non-motorized modes, namely bicycling and walking. We note that difficulties in calculating accessibility measures arise primarily from problems with data quality, the zonal structure of transportation planning models, and the adequacy of models and travel networks for describing and predicting travel by non-motorized modes. We present practical strategies for addressing these issues. The application of these methods is illustrated with the calculation of accessibility measures for a small study area in Minneapolis, MN (USA). The paper concludes with lessons learned from the process of developing non-motorized accessibility measures and ideas about their applicability to the practice of transportation planning.
\end{abstract}

Keywords: Pedestrian; Bicycling; Accessibility; Travel Behavior 


\section{Introduction}

Accessibility has been a well-known concept in the transportation planning field since the 1950s, when it was introduced as ease of reaching desirable destinations (Hansen 1959) tying land use and activity systems with the transportation networks that serve them. Improving accessibility has recently re-emerged as a central aim of urban planners and aligned disciplines. However, conventional transportation planning has often focused on improving movement (or mobility)—most often by the automobile. To the extent that accessibility has been measured or used in transportation planning, such measures have also been auto-based. In addition, they have typically only focused on access to employment.

The emphasis on employment accessibility is understandable, given its link to other important aspects of urban structure, such as choice of residential location, and also to outcomes hypothesized to be related to urban structure, such as social exclusion (Preston and Raje 2007). However, access to other types of destinations, such as retail, are also important because they strongly influence various dimensions of travel behavior such as trip frequency (Daly 1997), destination choice (Handy 1993), mode choice, and trip or tour complexity (Hanson and Schwab 1987).

Broadening the scope of accessibility to include additional types of destinations and nonauto modes such as walking and cycling has been long talked about in land use-transportation discussions (Handy 1993; Handy and Clifton 2001). To date, however, such discussions have been short on execution. Issues including, but certainly not limited to lack of reliable data, computational power or knowledge of non-motorized travel behavior have prevented widespread application of such measures.

This paper discusses such hurdles, presents alternatives for circumventing them, and demonstrates how accessibility for walking and cycling-and for different types of 
destinations—can be reliably measured. We focus on explaining specific features of nonmotorized transportation that complicate developing accessibility measures. These are discussed in the context of two major components of traditional accessibility measures: measures of destination attractiveness and travel impedance. The development of these components is illustrated with a sample application in Minneapolis, Minnesota, USA.

\section{Measuring Accessibility for Non-Motorized Travel}

In principle, it is logical to measure accessibility for non-motorized modes using similar methods as for motorized vehicle travel, thereby allowing the user to calculate any of the conventional measures of accessibility associated with zone-based travel forecasting models (e.g., cumulative opportunities, gravity-based, and utility-based measures). The measures most often used are gravity-based measures, in part due to their relative ease of calculation and interpretation. Gravity-based measures are derived from the denominator of the gravity model (Ingram 1971) and can be described with the general form:

\section{$A_{i}=\Sigma_{i} a_{i} f\left(t_{t i}\right)$}

...where $A_{i}$ represents accessibility at zone $i, a_{j}$ represents activity in zone $j$, and $t_{i j}$ represents travel impedance between $i$ and $j$, which can be expressed at time, distance, or cost, and $f\left(t_{i j}\right)$ is a function of $t_{i j}$ introduced to express the dampening effect of separation or cost on travel. Thus, accessibility reduces to a function of the size or availability of activities in each zone and the cost of accessing those activities.

Calculating gravity-based and other conventional, zone-based measures of accessibility benefits from compatibility with regional travel forecasting models which can easily extract zone-to-zone travel times from coded networks. In addition, counts of potential opportunities 
such as employment are stored at the zone level. Extending this basic framework to measure non-motorized travel encounters some serious limitations, however.

With respect to travel impedance, the networks used for modeling vehicular flows are too coarse to represent the route choices typically exercised by pedestrians and bicyclists. Also, the zones of these models are poorly matched to the spatial scale of movement by these modes, resulting in a considerable number of intrazonal trips (Eash 1999). While vehicular travel tends to be most sensitive to travel times and levels of network congestion, non-motorized route choices tend to include factors that may be more qualitative, experiential or difficult to operationalize, such as facility design and aesthetic treatments that may fall under the broad category of “environmental factors” (Porter et al. 1999, Tilahun 2007). That is not to suggest travel time is not an important determinant of route choice for non-motorized travelers (Stinson and Bhat 2003; Weinstein 2007)_ _ just that it is not quite as decisive.

Measures of attractiveness that influence the destination of trips (and hence accessibility) have traditionally been dealt with by intentionally simplifying a more complex problem. Models describing the choice among competing destination locations have typically treated geographically convenient units (such as travel analysis zones) as distinct alternatives, differentiated by some proxy measure of activity, such as retail or non-retail employment. Realworld travel choices are rarely this simple, and researchers interested in consumer behavior as an example of spatial choice have uncovered a number of attributes contributing to the choice of shopping location in addition to travel distance, such as prices, the quality and variety of goods, and the availability of parking.

In addition to these intrinsic attributes, factors such as historical dependence or brand loyalty have also been shown to influence store choices during shopping trips (Miller and 
O’Kelly 1983). Since many store attributes are difficult to measure in any great detail for modeling purposes, some researchers have chosen alternative measurement techniques, such as psychometric approaches (Recker and Kostyniuk 1978; Louviere 1979) and stated preference designs (Timmermans 1996). Calculation accessibility measures, however, requires the feasible collection of objective data on suitable measures of attractiveness. Ideally, this requires collecting data with more detail than simple counts of employment or floor space, and more realism than abstract or aspatial, hypothetical choice situations.

\section{Why Is It So Difficult? The Nuts and Bolts}

Having discussed some of the central parameters to measure access, generally, as well as access for non-motorized modes, we now turn to addressing specific sources of difficulty encountered with the inputs to accessibility calculations, namely issues stemming from data, zonal structure, networks, and behavior.

\subsection{Data}

Calculating accessibility measures requires multiple data sets relating to travel behavior and land use, each of which presents unique challenges for analysts addressing non-motorized modes. For example, robust accessibility measures are built around models representing human behavior (e.g., who shops where and how far they travel for such). Unfortunately, the data necessary to reliably build such models is in short supply for walking and cycling. User and trip characteristics at a suitable level of aggregation, along with user preferences for facility design characteristics are currently of limited quality and are considered a high priority for improvement (USDOT 2000). These data items are not adequately covered in most large scale survey 
instruments, such as metropolitan travel surveys or the Nationwide Personal Transportation Survey (NPTS).

Such issues often result in analysts borrowing assumptions from analysis usually slated for other purposes. A common example is an analysis borrowing impedance values from a locally-calibrated travel model. The values extracted from these data may be sensitive to the environment in which they were collected. Ideally, travel survey data would be collected year round and cover all seasons (Ortuzar and Willumsen 2001). More commonly, data are collected over a period of several months and reflect weather conditions prevailing at the time the survey data were collected. This is especially important in the case of non-motorized modes and in locations where significant seasonal climate variations exist. For example, if survey data are collected during warmer, drier months it is possible that changes in travel behavior during colder or more precipitous months might be missed. These changes might include mode shifts, in which case the number of pedestrians and bicyclists might be overestimated during cold weather periods, and changes in destination choice for discretionary trips, which would affect the length or distance of travel, and hence the relevant impedance values.

The quality of land use data also affects the accuracy of accessibility measures. Extending the range of desired destinations beyond employment and improving the accuracy or robustness of accessibility calculations requires data at a spatial resolution that is not typically available in most planning organizations. There are sources of establishment-level data on attributes such as employment, sales and other variables that could potentially serve as good proxy variables for attractiveness and be easily scaled to different levels of geographic aggregation. However, these sources are typically private financial organizations or highly confidential. The data can be costly to acquire and require significant effort in terms of cleaning 
and preparation for spatial analytical use. Alternate, low-cost sources of data such as business directory telephone listings have been employed elsewhere (Handy and Clifton 2001) in the context of the calculation of measures of "neighborhood” accessibility, though these data sets apparently contain limited information on size or quality of establishments.

\subsection{Zonal structure}

In addition, other efforts often use zones as units of analysis that do little justice to the detailed nature of pedestrian travel. For example, they may aggregate information to census tracts, zip code areas or TAZs. These units often do little justice to the central aim; they can be quite large, almost two miles wide and contain over 1000 households. The problem is that an ecological fallacy arises because average demographic or urban form characteristics are assumed to apply to any given individual neighborhood resident. When measures of commercial intensity are aggregated, for example, each zone reveals the same measure despite each zone exhibiting considerably different development patterns. Using census tracts or TAZs, concentrations of development may be averaged with adjacent lower-density development thereby making it difficult to associate many neighborhood-scale aspects with travel demand. The heart of the problem — and the ability to detect such subtle geographical differences-lies with the size of the units of analysis that are employed.

\subsection{Inadequate Networks}

Networks employed for purposes of regional travel models typically replicate roadways. Networks for walking and cycling are often different and need to be drawn at a finer scale. Specifically, the network structure is too coarse to trace the paths chosen by pedestrians and cyclists, and the zones are too large to differentiate many of the shorter trips made by bicycle and 
on foot. Also, few networks contain links with specialized facilities for non-motorized travel, such as sidewalks, exclusive bike paths and on-street bicycle lanes.

One way around these problems, as will be described in greater detail in a later section, is to use street network layers encoded as geographic information system (GIS) files as the basis for calculation of a minimum-cost path (with distance as a proxy measure for cost) between an origin and destination point, assuming agreement between the minimum-cost path and the actual chosen path (Witlox 2007). These networks can be manually modified to include certain types of special facilities. However, few cities or regional authorities have complete inventories of pedestrian and bicycle facilities, making the construction of a complete pedestrian and bicycle network a resource-intensive task. If time is the desired impedance measure, then assumptions need to be made about the relationship between distance and time in terms of an average speed. While this may be acceptable for pedestrian travel, the availability of bicycle facilities may alter bicyclists' travel time, necessitating special treatment of these facilities (El-Geneidy et al. 2007).

\subsection{Inadequate Models}

Related to the issue of inadequate networks and data is the applicability of model components of four-step transportation planning models to non-motorized modes. Most relevant to accessibility calculations is the impedance function, representing the influence of travel time, money and other costs on the willingness of individuals to travel longer distances. In transportation planning practice, it has been common to use gravity or other synthetic models to forecast the spatial distribution of trips, from which an impedance value can be estimated. While this approach works reasonably well for motorized modes, which tend to have a more regional distribution, there are often a large number origin-destination pairs with zero observations. This problem, known as the sparse matrix problem, is exacerbated by the application of such models 
to origin-destination data for non-motorized modes, which tend to have a more concentrated spatial distribution.

Since the full specification of the gravity model is not applicable for forecasting the distribution of trips by non-motorized modes over a large area, some modifications must be made. One option is to estimate impedance directly from the frequency distribution of trip lengths. While this approach is feasible, it has some serious limitations. Estimating an impedance parameter in the absence of information about the spatial distribution of activities (as is provided in the gravity model) is equivalent to assuming that activities are evenly distributed in space (Sheppard 1995). Clearly this assumption is not reasonable for most metropolitan regions and can lead to biased results.

A second caveat relates to the functional form of the impedance function. While many different specifications of the impedance function have been used, there is little available evidence to suggest a priori which one might be superior. Most of the specifications differ in their treatment of the effects of distance, which would in turn affect accessibility measurement. The simplest option would be to use the basic Newtonian gravity relationship $\left(1 / t^{2}\right)$, where $t$ represents travel time. A second option is to use the negative power function $\left(c_{i j}{ }^{-\beta}\right)$, where $c_{i j}$ represents the cost of travel between zones $i$ and $j$. This form was commonly used in some earlier transportation planning studies (1971). A third option, and one most commonly chosen, is the negative exponential form $\left(e^{-\beta x}\right)$. This function has the advantage that it declines more gradually than the power function, and thus better estimates shorter trips (Kanafani 1983). This advantage, along with a record of numerous empirical applications made it an appropriate functional form to be estimated for the set of impedance functions applied in the current study. 
In addition to choosing a form for the impedance function, the analyst must specify which variable is being used to measure separation or impedance (time, cost or both). In practice, both measures have been used, along with some examples of the use of the generalized cost concept (Handy and Niemeier 1997). In the case of non-motorized travel, however, the options appear to be limited to the use of distance, due to the problems associated with extracting accurate travel times from existing network models for bicycling and walking.

\section{Coping With Limitations: How It Can Be Done}

The previous section outlined many of the hurdles in developing measures of nonmotorized accessibility. This section describes strategies to overcome these hurdles and uncover

robust measures of non-motorized access for urban areas. These strategies are fourfold, including (1) addressing the network and zonal aggregation issues using block-level data and special networks, (2) preparing detailed land use data, (3) collecting appropriate travel survey data, and (4) developing impedance measures. Some results of a small-scale application of these measures to a portion of south Minneapolis, Minnesota (USA) better illustrates the process.

\subsection{New Networks and Zones}

Previously, attention was paid to the issue of incompatibility between conventional travel forecasting models and travel by non-motorized modes. Travel zones are too large and networks too coarse to provide detailed analysis of destination and route choice behavior by pedestrians and bicyclists. This is one area where compromise solutions must be adopted in order to make the research problem tractable.

The task of calculating travel times via a network model is one that is not easily resolved. The only apparent alternative to using the networks in existing travel models is to use street 
networks encoded as GIS files to measure minimum-cost paths between geocoded origin and destination locations. This method ignores the matter of congestion on networks, since it is impossible to code an entire network with the appropriate capacity data. However, many studies of accessibility choose to ignore congestion effects and simply use free-flow travel times as a reasonable approximation.

GIS networks can be manually modified in order to incorporate the presence of special facilities, such as exclusive bicycle paths or joint use bike/pedestrian paths. In principle, these links are chosen because they offer travel time, quality or other advantages that lower the perceived “cost” of travel by non-motorized modes. These advantages can be operationalized by giving these links a lower cost than other unimproved links. If data on exclusive pedestrian and bicycle facilities are not available in a digital format, they can be checked against published maps or other available sources. This method was applied to the Twin Cities' network of exclusive bicycle paths, which were recreated from a locally published bicycle system map.

A key assumption of constant travel speeds must be accepted for bicycle and pedestrian travel, in order for this method to be applicable. This allows for simple conversions between measurement of distance and time. As a check on this assumption, El-Geneidy and others (2007) reviewed the literature on travel speeds for pedestrian and bicycle modes and tested the influence of different types of bicycle facilities on travel speeds. Off-street facilities were shown to have a small but significant effect on speeds, lending support to their inclusion as special network links with different cost characteristics. However, this work also noted a high degree of interpersonal variability, indicating that an assumption of constant speeds may be a significant source of uncertainty in accessibility measures. 
Another adaptation that allows a better characterization of travel impedance is using smaller zones to identify potential origins and destinations. This method has been used elsewhere (Eash 1999) to model non-motorized destination choice, using zones roughly aligned with Census tracts. An alternative-and smaller-zone designation used in the Twin Cities application is to use grid cells or Census block groups, which are similar in size and function.

\subsection{Detailed Land Use Data}

Developing measures of attractiveness at a more detailed level than the zones used in travel forecasting models requires specialized, establishment-level data that can be aggregated to the level of small units, such as the block groups described earlier. Establishment-level data was purchased from Dun \& Bradstreet, Inc. containing attribute information on location, sales, employees, and industry classification. In all, data were available for 135928 businesses within the region. These data were merged with parcel-level land use data from the Metropolitan Council, the Twin Cities’ regional planning agency. The establishment-level data were then recoded into destination categories using the 2 to 6-digit classifications of the North American Industry Classification System (NAICS). The outcome of this process was a set of parcel-level land use data with information on employment counts and sales volumes. A small sample of this data set, with mapped parcel-level land use for an eight-block area of south Minneapolis, is shown in Figure 1.

[Place Figure 1 here]

\subsection{Travel Survey Data}

Estimating specialized impedance functions specific to non-motorized modes requires appropriate travel survey data that can capture pedestrian and bicycling behavior. Ideally, this 
would involve a focused, special-purpose survey designed to oversample these types of behavior. In the absence of such data, a regional household travel survey can be used to the extent that it includes trips by non-motorized modes. A limitation of this approach, however, is the variety of destinations that can feasibly be studied. Given that walking and bicycling tend to be seldomused and often underreported modes, any further partitioning of the data can lead to small samples and less robust inferences.

\subsection{Choice and Estimation of Impedance Measures}

One of the critical steps in developing accessibility measures is designating impedance values to represent the friction of travel to various destinations. Past research has suggested that using either time or distance as an impedance variable is acceptable (Handy and Niemeier 1997), though very detailed and data-rich applications might use the logsum of the mode choice calculation for a given origin-destination pair. Intrinsically, time is an appealing measure, since it represents a scarce resource that must be expended during travel. It also carries the advantage of widespread application in other areas of travel modeling. At the same time, the methods outlined here for approaching the problem of dealing with non-motorized travel suggest that distance data may be easier to obtain.

To resolve the matter of which impedance variable to use, both were tested in the calculation of accessibility measures and compared. Gravity-based accessibility measures were calculated for work, shopping and restaurant trips by walking and bicycling modes using time and distance variables. Simple correlation coefficients between the time and distance-based measures ranged from approximately 0.92 to just under one, indicating little sensitivity to the specification of impedance variable. 
To calculate impedance values for each mode and trip purpose, household travel survey data was used to fit a negative exponential curve that provided a continuous approximation to the shape of the trip length distribution, using both trip duration and distance data. The same functional form was used for all impedances to ensure consistency of application across modes and trip purposes. A set of impedance functions for walk trips using distance as an impedance measure is provided in Figure 2. Destinations for which these functions were estimated include work, shopping, restaurant and entertainment trips. The full summary of impedance functions for walking and bicycling is shown in Table 1.

[Place Figure 2 about here]

[Place Table 1 about here]

One drawback of this method is that it imposes the same functional form on each impedance function regardless of the underlying distribution, thus producing a poor fit in some situations. Another important issue is that the impedance functions are estimated without reference to the spatial distribution of activities, meaning that the estimated impedance parameter may mask a significant amount of variation between geographic locations. Nonetheless, this procedure provides a disaggregate alternative to assuming identical travel behavior for all trip purposes.

\section{An Example of Accessibility Measures}

To illustrate the procedures used to produce estimates of non-motorized accessibility, we completed some sample accessibility calculations for a small study area in South Minneapolis ${ }^{3}$.

\footnotetext{
${ }^{3}$ The study area is bounded on the west by Lyndale Avenue, on the north by Franklin Avenue, on the east by the Mississippi River, and on the south by $50^{\text {th }}$ Street.
} 
This area contains approximately 1600 block groups, which represent the unit of analysis. The accessibility values calculated for each block group are integral accessibility measures (Song 1996), where the activities in each destination zone, discounted by their associated impedance value, are summed across destinations and normalized by dividing by the total activities in the study area. This method provides a measure that can be easily interpreted and compared across zones on the same zero to one scale. Analytically, this measure is represented as

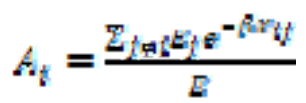

where:

$A_{i}$ denotes accessibility evaluated at origin zone $i$

$x_{i j}$ denotes the distance (or travel time) between zones $i$ and $j$

$E_{j}$ denotes the amount of activity in destination zone $j$

$E$ denotes total activity in the study area, summed across all zones, and

$\beta$ is a parameter of the impedance function, to be empirically estimated

Thus, in each case, accessibility is expressed as a decimal indicating proximity to destinations in each location. In the case of each accessibility calculation, an attractiveness measure is constructed for each block group by summing the level of retail sales at each establishment within the block group. Impedance measures are introduced by calculating the shortest path through the network between each block group pair, then using this value to discount activities at the destination using the functional form described previously.

Figure 3 presents maps displaying measures of accessibility to restaurant destinations for the walking mode. Again, the maps show the same measures calculated using time and distance as alternate measures of travel impedance. Consistent with the findings described earlier, they 
show a high degree of correlation. Areas near clusters of restaurant destinations are shown to have high levels of accessibility, with a gradual decline as one moves away from these clusters.

[Insert Figure 3 here]

Figure 4 presents a pair of maps showing accessibility to shopping destinations by bicycle with distance and time impedance measures. In this case, destinations are spread more evenly throughout the study area, leading to higher overall accessibility values in each zone. Retail establishments appear to align themselves along linear corridors, reflecting the historical network of streetcar routes in South Minneapolis. One particularly large corridor is found along Lake Street, a major east-west route that lies at the center of the swath of high accessibility shown in both maps. This high-accessibility location results from a combination of clustering of activities and proximity to the Midtown Greenway, a grade separated off-street bicycle facility highlighted in green on the map.

[Insert Figure 4 here]

Together, these two examples illustrate the roles that location and space play in determining non-motorized accessibility, robustly measured, for an urban area, and graphically displays the outcomes associated with the interaction of these forces.

\section{CONCLUSIONS AND LESSONS LEARNED}

Several points are important from this research that relate to designing methods for calculating non-motorized accessibility measures. Each of these will be discussed in turn in this concluding section.

First, we have shown that it is in fact possible to construct meaningful measures of accessibility for non-motorized modes. This effort has gone beyond previous work in this area 
by attempting to introduce more behavioral realism into accessibility calculations and doing so for relatively small units of analysis. Such realism is accomplished primarily through the use of impedance measures estimated for each separate combination of mode and trip purpose and highly detailed land use data. This represents an improvement over previous studies, which often borrowed values from other studies or relied on assumptions about the true value or aggregate values for a large area. Furthermore, the estimation of the impedance measures was aided by the use of a specially-constructed network that was designed to capture a fuller range of route choices for pedestrians and cyclists than most travel model networks allow.

Second, there are real tradeoffs in developing non-motorized accessibility measures. One is that the work required to develop these types of measures is resource-intensive. The time and money costs required to collect the needed data and conduct the analysis are seldom found outside of large research budgets. Only the largest planning organizations would be likely to have the personnel and financial resources to replicate the work described here. Among these requirements would be the need to collect special travel survey data catered to the behavior of pedestrians and cyclists. The only alternative to this, using general-purpose travel survey data, was employed in the current study. This practice led to difficulties in accommodating multiple destination types due to limited sample sizes.

A further tradeoff is that the methods presently described for calculating non-motorized accessibility involve multiple sources of uncertainty. A short list of these sources might include, but are by no means limited to

- Assumptions about average travel speeds by pedestrians and cyclists

- Calculation of travel distances by a minimum-cost path method that may or may not be appropriate for non-motorized travel 
- Impedance functions for mode and trip purpose, many of which were estimated based on small samples and so include high variances

- Measurement of attractiveness by retail sales. This method, while probably an improvement over simple counts of employment, does not distinguish between shopping centers involving several retailers versus a single large, multipurpose (“big box”) retailer

- The lack of agglomeration effects in attractiveness measures due to the use of small analysis units such as block groups, which may be exceeded by the extent of large retail centers

Some of these sources of uncertainty may be accounted for through sensitivity analysis or simulation methods; others present serious measurement problems and must be accepted as caveats to the validity of the results.

It may be that a high degree of accuracy is not required of these measures, though. Rather, their value may lie in informing the design of instruments of accessibility-related policies (Farrington 2007), scenario building and sketch planning applications. For example, the maps in Figure 3 indicate that there are large portions of the study area with relatively low walk accessibility to restaurants. This finding might prompt efforts to reduce zoning restrictions in certain neighborhoods to allow new restaurants to locate in underserved areas. Or perhaps it may indicate that improvements to the pedestrian infrastructure are warranted. Either approach could be employed to address the stated goal of improving access. In addition to formulating planning goals, non-motorized accessibility measures can provide one important component of an overall system for monitoring and evaluating the transportation and land use system in an urban region. With a growing level of interest in non-motorized travel in many transportation policy circles, 
detailed and robust accessibility measures geared to non-motorized modes provide an additional option to form and evaluate land use-transportation planning efforts.

\section{Acknowledgements}

The authors thank Ryan Wilson, Jessica Horning and Gavin Poindexter for assisting in creating the networks, cleaning the parcel data, and preparing the calculations necessary for this work.

\section{References}

Daly, A.J., 1997. Improved methods for trip generation. In: Proceedings of the $25^{\text {th }}$ European Transport Conference, Vol. 2. London: PTRC, pp. 207-222.

Eash, R.W., 1999. Destination and mode choice models for nonmotorized travel. Transportation Research Record 1674, 1-8.

El-Geneidy, A.M., Krizek, K.J., Iacono, M.J., 2007. Predicting bicycle travel speeds along different facilities using GPS data: a proof of concept model. In Proceedings of the $86^{\text {th }}$ Annual Meeting of the Transportation Research Board, Compendium of Papers. CD-ROM. TRB: Washington, D.C.

Farrington, J.H., 2007. The new narrative of accessibility: its potential contribution to discourses in (transport) geography. Journal of Transport Geography 15(5), 319-330.

Handy, S.L., 1993. Regional versus local accessibility - implications for nonwork travel. Transportation Research Record 1400, 58-66.

Handy, S.L. \& Clifton, K.J., 2001. Evaluating neighborhood accessibility: possibilities and practicalities. Journal of Transportation and Statistics 4(2/3), 67-78.

Handy, S.L. \& Niemeier, D.A., 1997. Measuring accessibility: an exploration of issues and alternatives. Environment and Planning 29A(7), 1175-1194.

Hansen, W. , 1959. How accessibility shapes land use. Journal of the American Institute of Planners 25(1), 73-76.

Hanson, S. \& Schwab, M., 1987. Accessibility and intraurban travel. Environment and Planning 19A(6), 735-748.Ingram, D.R., 1971. The concept of accessibility: a search for an operational form. Regional Studies 5, 101-107.

Kanafani, A.K., 1983. Transportation Demand Analysis. New York: McGraw-Hill. 
Louviere, J.J., 1979. Applications of psychological measurement and modelling to behavioural travel-demand analysis. In D.A. Hensher \& P.R. Stopher, eds. Behavioural Travel Modelling. London: Croom Helm. pp. 713-738.

Miller, E.J. \& O'Kelly, M.E., 1983. Estimating shopping destination choice models from travel diary data. Professional Geographer 35(4), 440-449.

Ortuzar, J. de D. \& Willumsen, L.G., 2001. Modelling Transport. $3^{\text {rd }}$ ed. New York: John Wiley and Sons, Inc.

Porter, C., Suhrbier, J.H., Schwartz, W.L. 1999. Forecasting bicycle and pedestrian travel: state of the practice and research needs. Transportation Research Record 1674, 94-101.

Preston, J. \& Raje, F., 2007. Accessibility, mobility and transport-related social exclusion. Journal of Transport Geography 15(3), 151-160.

Recker, W.W. \& Kostyniuk, L.P., 1978. Factors influencing destination choice for the urban grocery shopping trip. Transportation 7(1), 19-33.

Sheppard, E., 1995. Modeling and predicting aggregate flows. In S. Hanson, ed. The Geography of Urban Transportation. $2^{\text {nd }}$ ed. New York: Guilford Press. pp. 100-128.

Song, S., 1996. Some tests of alternative accessibility measures: a population density approach. Land Economics 72(4), 474-482.

Stinson, M.A. \& Bhat, C.R., 2003. Commuter bicyclist route choice: analysis using a stated preference survey. Transportation Research Record 1828, 107-115.

Tilahun, N.Y., Levinson, D.M., Krizek, K.J., 2007. Trails, lanes, or traffic: valuing bicycle facilities with an adaptive stated preference survey. Transportation Research, Part A: Policy and Practice 41A(4), 287-301.

Timmermans, H.J.P., 1996. A stated choice model of sequential mode and destination choice behavior for shopping trips. Environment and Planning 28A(1), 173-184.

U.S. Department of Transportation, Bureau of Transportation Statistics., 2000. Bicycle and Pedestrian Data: Sources, Needs, \& Gaps. Publication BTS00-02. Washington, D.C.: U.S. Department of Transportation.

Weinstein, A., Bekkouche, V., Irvin, K., Schlossberg, M., 2007. How far, by which route, and why? A spatial analysis of pedestrian preference. In Proceedings of the $86^{\text {th }}$ Annual Meeting of the Transportation Research Board, Washington, D.C., USA, 21-25 January 2007. TRB:

Washington, D.C.

Witlox, F., 2007. Evaluating the reliability of reported distance data in urban travel behavior analysis. Journal of Transport Geography 15(3), 172-183. 
Table 1: Summary of impedance functions for walking and bicycling

\begin{tabular}{|c|c|c|c|c|c|c|c|c|c|c|}
\hline & \multicolumn{2}{|c|}{ Work } & \multicolumn{2}{|c|}{ Shopping } & \multicolumn{2}{|c|}{ School } & \multicolumn{2}{|c|}{ Restaurant } & \multicolumn{2}{|c|}{ Recreation } \\
\hline & Distance & Time & Distance & Time & Distance & Time & Distance & Time & Distance & Time \\
\hline Walk & $y=.486 e^{-1.683 x}$ & $\mathrm{y}=.511 \mathrm{e}^{-.106 x}$ & $y=.469 e^{-2.106 x}$ & $\mathrm{y}=.368 \mathrm{e}^{-.094 \mathrm{x}}$ & & $y=.524 \mathrm{e}^{-.106 \mathrm{x}}$ & $\mathrm{y}=.388 \mathrm{e}^{-1.397 \mathrm{x}}$ & $y=.373 e^{-.093 x}$ & $\mathrm{y}=.327 \mathrm{e}^{-.769 \mathrm{x}}$ & $\mathrm{y}=.556 \mathrm{e}^{-.100 \mathrm{x}}$ \\
\hline Bike & $y=.402 e^{-.203 x}$ & $\mathrm{y}=.146 \mathrm{e}^{-.040 \mathrm{x}}$ & $\mathrm{y}=.343 \mathrm{e}^{-.514 \mathrm{x}}$ & $\mathrm{y}=.434 \mathrm{e}^{-.107 \mathrm{x}}$ & $\mathrm{y}=.458 \mathrm{e}^{-.122 \mathrm{x}}$ & $\mathrm{y}=.424 \mathrm{e}^{-.100 \mathrm{x}}$ & & & $\mathrm{y}=.367 \mathrm{e}^{-.375 \mathrm{x}}$ & $\mathrm{y}=.293 \mathrm{e}^{-.071 \mathrm{x}}$ \\
\hline Notes: & & & & & & & & & & \\
\hline
\end{tabular}




\section{Figure 1: Parcel-level land use data}

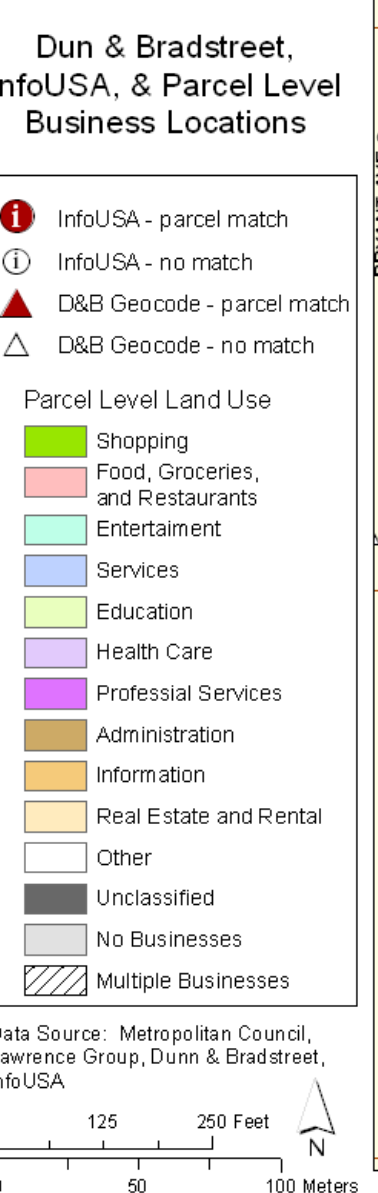

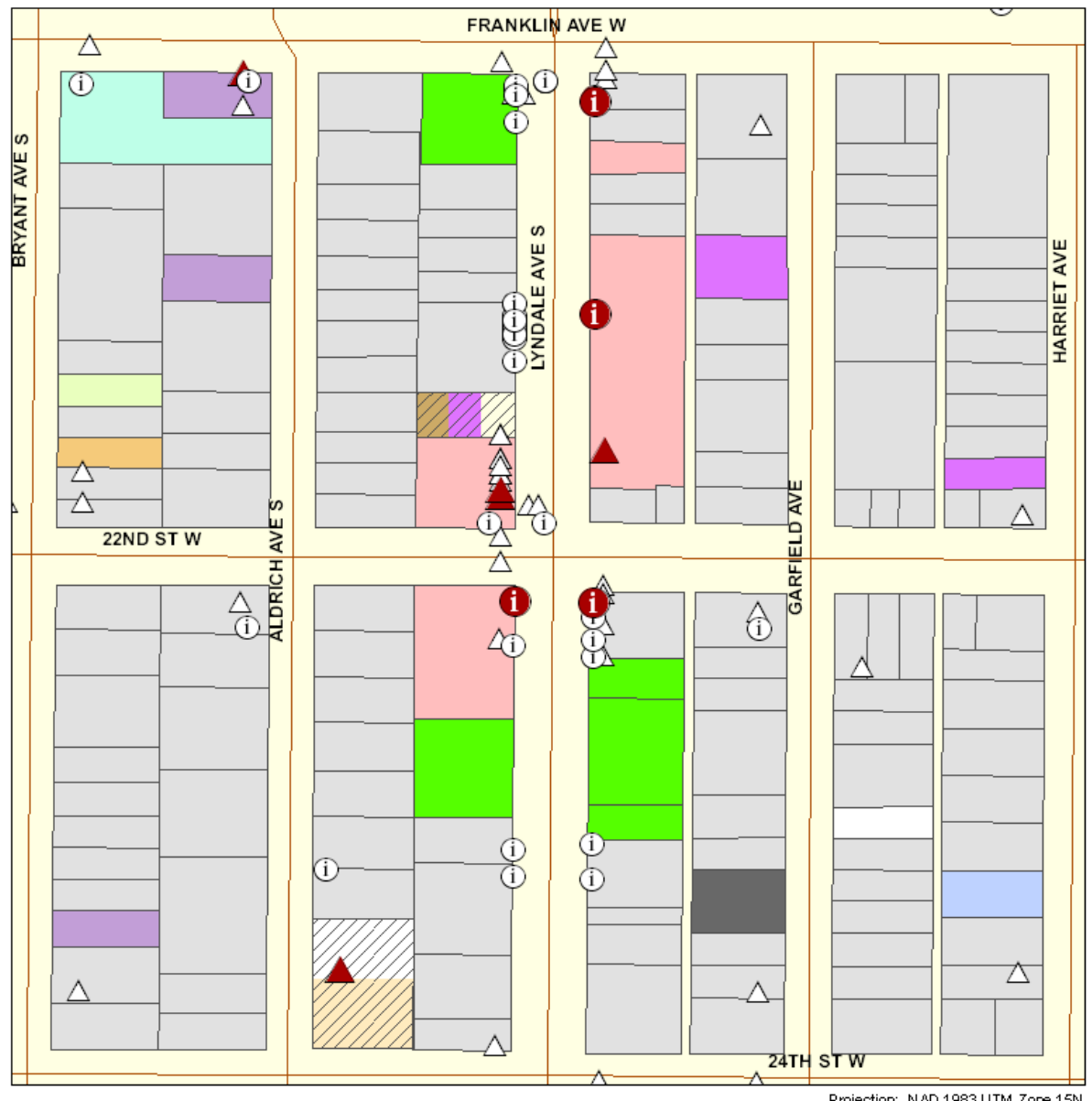


Figure 2: Impedance functions for walking trips

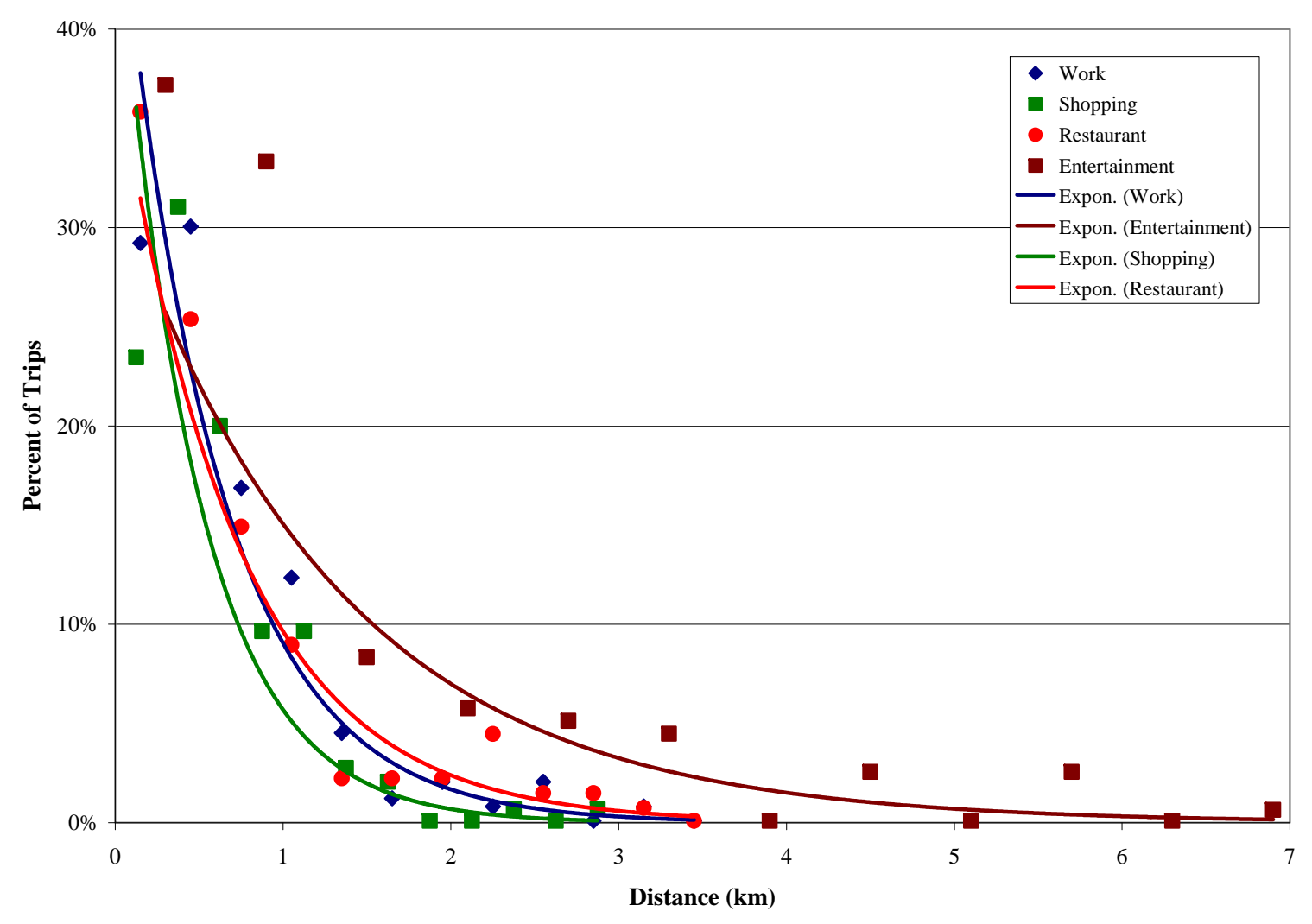


Figure 3: Walk accessibility to restaurants

Walking

Integral Accessibility to Restaurant Destinations: Using Distance \& Time Impedance Parameters

\begin{tabular}{|c|}
\hline Integral Accessibility \\
\hline \\
\hline \\
$0.0025-0.0028$ \\
$0.0021-0.0024$ \\
$0.0017-0.0020$ \\
$0.0013-0.0016$ \\
$0.0009-0.0012$ \\
$0.0005-0.0008$ \\
$\quad 0.0000-0.0004$ \\
Restaurants \\
Major Roads \\
\hline Interstate \\
\hline State Highway \\
\hline US Highway \\
Parks \\
Water
\end{tabular}

Data Source: Metropolitan Council, Lawrence Group, Dunn \& Bradstreet,

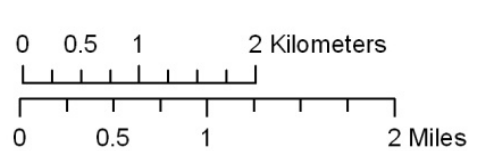

Projection: GCS North America 1983
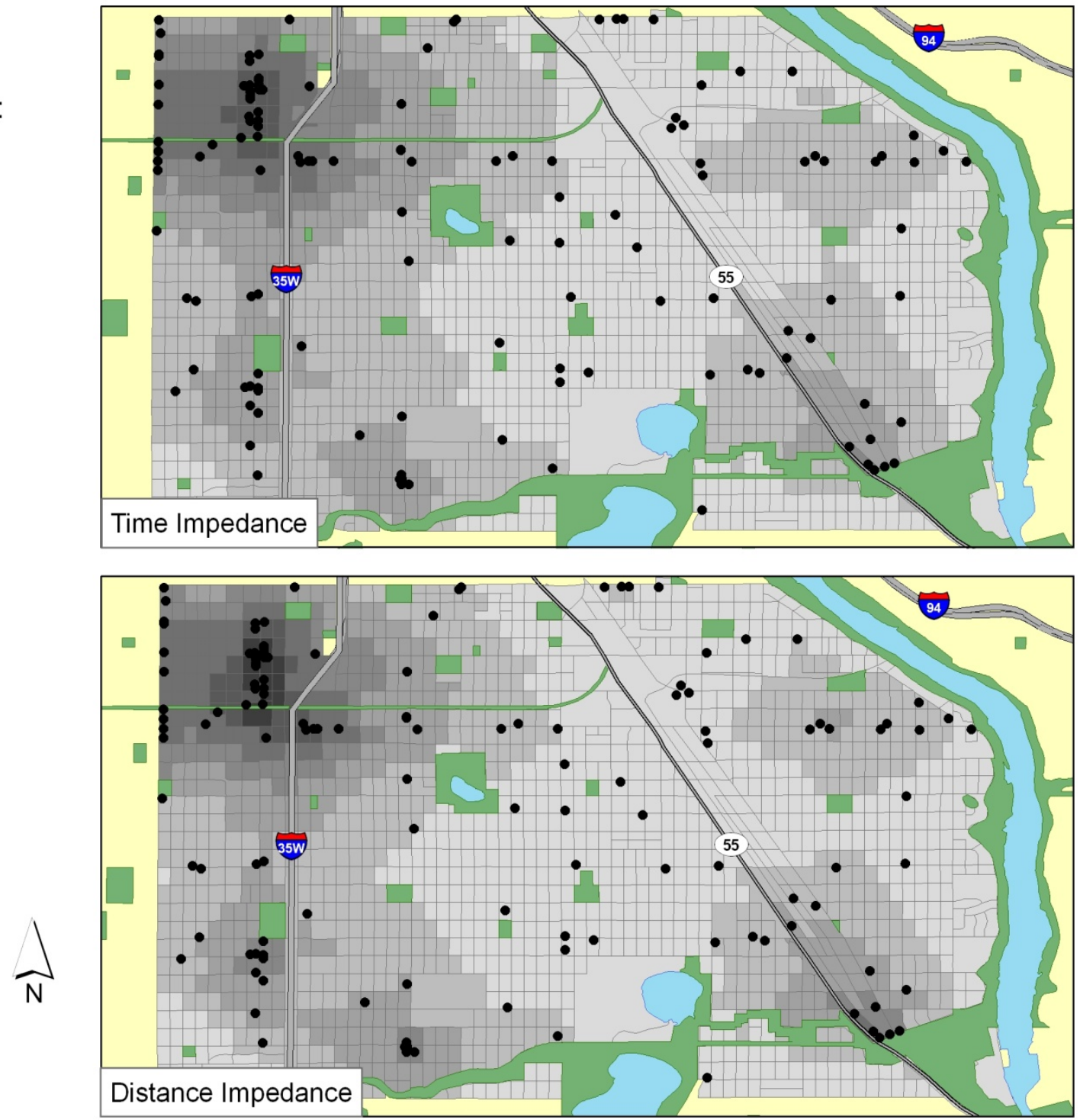


\section{Figure 4: Bicycle accessibility to shopping}

Bicycling

Integral Accessibility to Shopping Destinations: Using Distance \& Time Impedance Parameters

\begin{tabular}{|c|}
\hline Integral Accessibility \\
\hline \\
$0.0012-0.0013$ \\
$0.0010-0.0011$ \\
$0.0008-0.0009$ \\
$0.0006-0.0007$ \\
$0.0004-0.0005$ \\
$0.0002-0.0003$ \\
$0.0000-0.0001$ \\
$\quad$ Shopping Store \\
Major Roads \\
\hline Interstate \\
\hline \hline
\end{tabular}

Data Source: Metropolitan Council, Lawrence Group, Dunn \& Bradstreet,

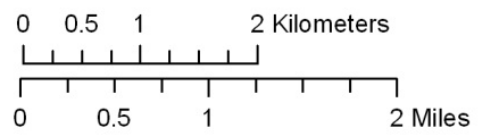

Projection: GCS North America 1983
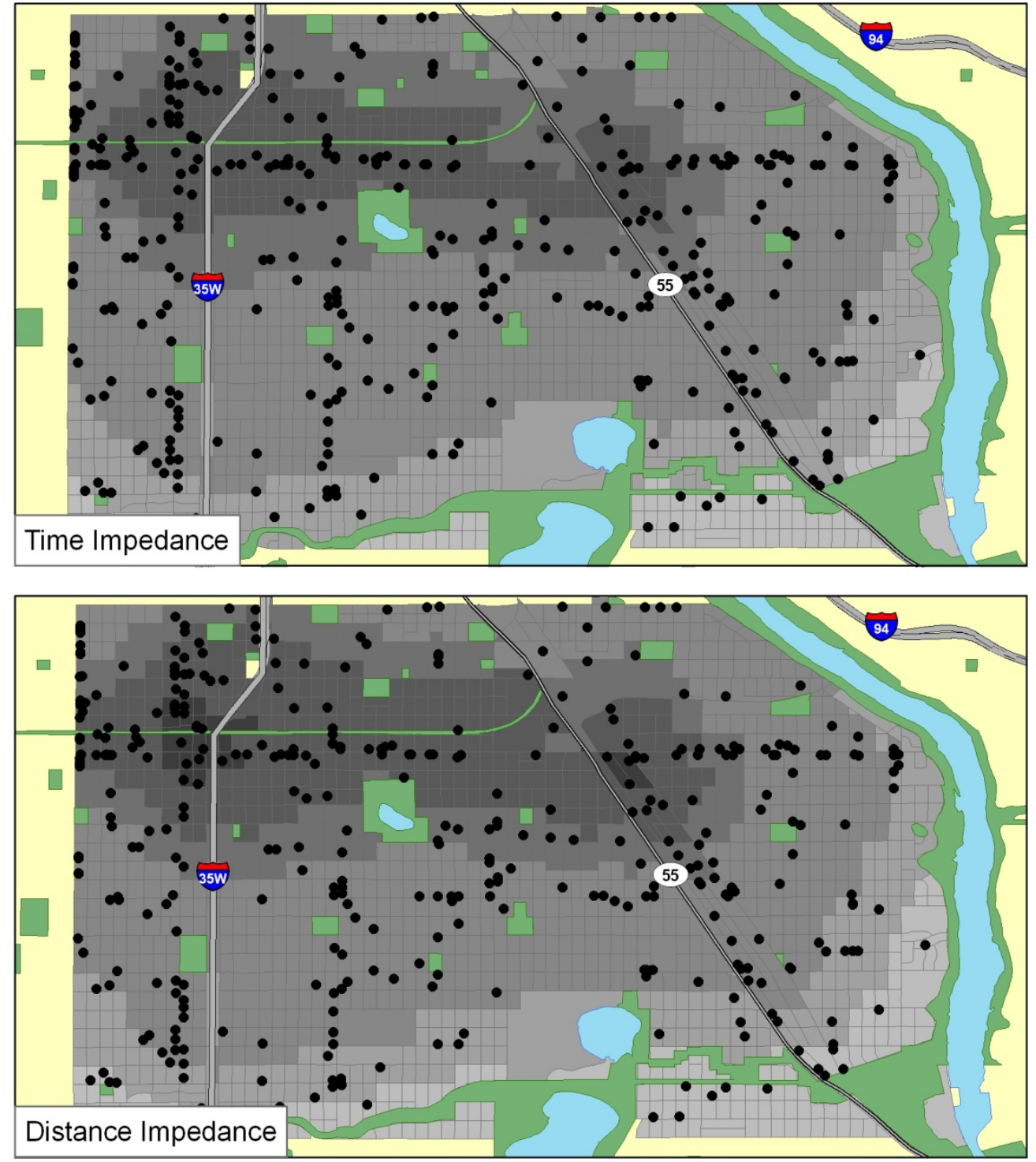